\title{
MENTAL HEALTH IN GENDER DYSPHORIA: IS COMORBIDITY ALARMING?
}

\section{Authors:}

T. Queirós (1), I. Souto Braz (1), C. Ferreira (1),

F. Semeão Coelho (1), R. Saraiva (1), J. Rema (1),

F. Santos (2), M. Melo (3), R. Barandas (1)

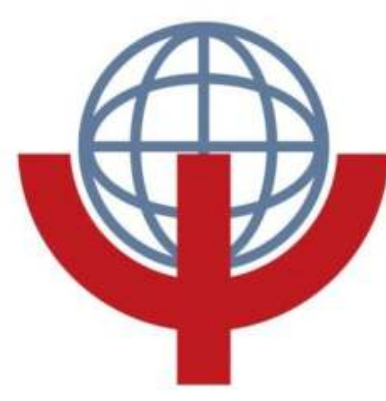

WORLD

PSYCHIATRIC

ASSOCIATION

(1) Centro Hospitalar Universitário Lisboa Norte, Serviço de Psiquiatria e Saúde Mental, Lisbon, Portugal. (2) Centro Hospitalar Universitário do Algarve, Serviço de Psiquiatria e Saúde Mental, Faro, Portugal. (3) Centro Hospitalar Psiquiátrico de Lisboa, Clínica 2, Lisbon, Portugal.

e-mail: tiago.queiros@chln.min-saude.pt

\section{OBJECTIVES}

Our objective is to examine current existing literature regarding common mental health issues and psychiatric comorbidity in individuals presenting with gender dysphoria (GD).

\section{BACKGROUND}

High rates of comorbid psychopathology are consistently reported in the literature. Mood and anxiety disorders are likely to occur in people with GD (lifetime prevalence of $60 \%$ and $28 \%$, respectively). There is still a lot of debate about this subject, especially regarding sex specificity.

\section{METHODS}

We surveyed all PubMed-available scientific articles up to March 2019 with the search term "gender dysphoria". Articles were chosen based on the topic of mental health in people with GD.

\section{RESULTS \& CONCLUSIONS}

Perceived prejudice and discrimination (often the result of transphobia and minority stress) have been found to be positively associated with an increased prevalence of mental health problems (current prevalence of $30-40 \%$ ). Mood and anxiety disorders are especially likely to occur in association with GD. It's important to stress that depression, suicidality and self-harm are more prevalent early in the transition process. Moreover, at the same time, people with GD tend to have an earlier onset of mental disorders.
This increased prevalence of mental disorders among transgender individuals may contribute to maladaptive strategies to cope with distress, including drug and alcohol abuse, sexual risk taking and self-injurious behaviours. Therefore, there is a major concern regarding suicidality and self-harm, since studies show that about 1 in 3 adults with GD has experienced these situations in their lifetime. Studies show that after sex reassignment surgery (SRS), people with GD were 2.8 times more likely than controls to have been hospitalized for a psychiatric problem other than GD (even after adjustment for prior psychiatric comorbidity). Also, after SRS these patients were 4.9 more likely to have made a suicide attempt and 19.1 times more likely to have died from suicide (again, after adjusting for prior psychiatric comorbidity).

Despite these concerning rates of comorbid psychopathology, studies show its significant reduction after the transition process, displaying the positive association with a successful transition (to initiate hormone treatment alone is associated with lower levels of psychopathology, better quality of life and mental wellness). Nonetheless, testosterone could have an activating effect on bipolar disorder (requiring close monitoring) and people on estrogen may experience more emotional intensity. More importantly, a greater social support was associated with less suicidality.

In recent years GD has been viewed as an identity rather than being perceived as a mental health disorder, reducing the associated stigma. Since mood and anxiety disorders are common in these patients, the clinician must be able to diagnose not only GD but also identify comorbid symptoms, especially before transition and treat them accordingly. Risk of suicidality remains alarming in these patients and support should be offered.

\section{References:}

- Zucker KJ., Lawrence AA., Kreukels BP. (2016), Gender dysphoria in adults in Annual Review of Clinical Psychology, 12, 217-247;

- Wanta JW., Unger CA. (2017), Review of the Transgender Literature: Where Do We Go from Here? in Transgender Health, 2(1), 119-128;

- Mueller SC., De Cuypere G., T'Sjoen G. (2017), Transgender Research in the 21st Century: A Selective Critical Review From a Neurocognitive Perspective in The American Journal of Psychiatry, 174(12), 1155-1162;

- Mizock L. (2017), Transgender and Gender Diverse Clients with Mental Disorders: Treatment Issues and Challenges in The Psychiatric Clinics of North America, 40(1), 29-39; 